\title{
Invasive Fungal Rhinosinusitis: An Observational Study in an Indian Tertiary Care Hospital
}

\author{
Ravinder Kaur $^{1^{*}}$, Lavanya $\mathbf{S}^{1}$, Nita Khurana ${ }^{2}$, Achal Gulati $^{3}$ and Megh Singh Dhakad ${ }^{1}$ \\ ${ }^{1}$ Department of Microbiology, Maulana Azad Medical College and Associated Hospitals, New Delhi, India \\ ${ }^{2}$ Department of Pathology, Maulana Azad Medical College and Associated Hospitals, New Delhi, India \\ ${ }^{3}$ Department of ENT, Maulana Azad Medical College and Associated Hospitals, New Delhi, India
}

*Corresponding author: Ravinder Kaur, Lady Hardinge Medical College and Associated Hospitals, New Delhi, India, Tel: 919811809868; E-mail: drkaur@hotmail.com Received date: December 22, 2015; Accepted date: May 19, 2016; Published date: May 26, 2016

Copyright: (c) 2016 Kaur R, et al. This is an open-access article distributed under the terms of the Creative Commons Attribution License, which permits unrestricted use, distribution, and reproduction in any medium, provided the original author and source are credited.

\begin{abstract}
Invasive fungal rhinosinusitis (IFRS) is a challenging condition and the mortality of IFRS in immuno compromised patients is very high. 75 patients of suspected FRS were included to study the burden and the clinicopathological and mycological profile of invasive fungal rhinosinusitis (FRS) in these patients. The samples collected were exudate from nasal debri, discharge and intraoperative tissue sample and were subjected to direct microscopy to histopathological examination and direct microscopy ( $\mathrm{KOH}$ and calcofluor white) and culture on Sabouraud dextrose agar. Identification of molds and yeasts were done by conventional methods. 25 cases were suspected to have IFRS were confirmed by microbiological and histo pathological examination, comprising $56 \%$ of AIFRS (acute IFRS), $36 \%$ CIFRS (chronic IFRS) and 8\% CGFRS (chronic granulomatous FRS). Rhizopus arrhizus (64.2\%) was the most common isolate followed by Aspergillus flavus (35.7\%). Mucor species were solely isolated from AIFRS (14.3\%). In CIFRS, Aspergillus flavus (44.4\%) seemed to be the major isolate with Aspergillus niger, Alternaria spp., Penicillium spp. and Candida albicans. Necrosis of submucosa, bone, vascular tissue was seen in $96 \%$ of cases with aseptate hyphae in $78.5 \%$ AIFRS and $77.7 \%$ in CIFRS. Radiological features help in presumptive diagnosis of FRS. Direct microscopy along with culture conformation is important for diagnosis and early initiation of treatment.
\end{abstract}

Keywords: Aspergillus; Fungal sinusitis; Invasive; Rhinosinusitis

\section{Introduction}

FRS is a known entity and has recently gained a lot of attention with increase in clinical suspicion, availability of a variety of diagnostic modalities and improvement in laboratory techniques for detection of fungi. Classification of FRS depending on the host immune interaction with the fungi and is important for effective treatment and understanding the prognosis of the disease [1]. Disease is most commonly classified as non-invasive or invasive depending on the invasion of the fungi into the sinonasal, submucosal tissue resulting in tissue necrosis and destruction [2-4].

IFRS is a challenging condition especially in immunocompromised patients. Failure to diagnose and treat this entity promptly usually results in rapid progression and death [5]. The mortality of IFRS in immunocompromised patients ranges from $50 \%$ to $80 \%$. And early physical findings are non-specific and ambiguous [6].

Diagnosis of FRS is based on a high index of clinical suspicion, clinical history and physical examination of the patient per se being rarely conclusive. Even though clinical presentation is very helpful for subcategorization of various types of FRS; the diagnosis finally depends upon direct microscopy, culture and histopathology. Direct microscopy and culture helps in establishing the aetiology [7], while radiological and histopathological pictures helps in differentiation of invasive and non-invasive types. Early diagnosis and optimum therapy go a long way in reducing the morbidity of these patients [8,9].

FRS is a common disorder in India, but scant in population based data. More studies are needed to understand the Indian occurrence and epidemiology of FRS [10]. Hence, a detailed examination along with microscopy and culture is necessary. An early and accurate diagnosis of various types of FRS is a must for working out an optimum treatment schedule of the patients $[4,10]$. The main objectives of this study were to assess the burden of IFRS in our hospital and to study the clinicopathological and mycological profile of the disease.

\section{Materials and Method}

\section{Design and setting}

Depending on the clinical presentation, nasal endoscopic and radiology pictures a total of 75 patients of suspected FRS from wards and OPDs of our hospital were included in this observational study over 2013-2014 after obtaining informed consent from them. Relevant clinical history, nasal endoscopy findings, radiological findings and treatment history were noted.

\section{Collection and processing of samples}

The samples collected were exudate from nasal debri, discharge and intraoperative tissue sample after obtaining informed consent from them. Samples were collected in two sterile containers, one containing normal saline and the other containing $10 \%$ formalin and processed in the Department of Microbiology and Pathology respectively. Samples received in Microbiology were subjected to direct microscopy using $\mathrm{KOH}$ and calcofluor white as well as culture onto two sets of tubes of Sabouraud dextrose agar (SDA) with and without antibiotics. Samples 
Page 2 of 7

sent in formalin to Pathology department were put up for histopathological examination (HPE).

\section{Identification of molds and yeasts}

Fungal growth was identified by colony morphology, Gram staining, LPCB (lactophenol cotton blue) preparation and Riddle's slide culture as per standard recommended procedures [11]. Identification of the yeasts was done on the basis of germ tube production, morphology corn meal agar and HiCrome agar morphology (Hi Media), carbohydrate fermentation tests using yeast nitrogen base agar ( $\mathrm{Hi}$ Media) as per standard recommended procedures [11-13].

\section{Statistical analysis}

SPSS software (version 17.0; SPSS S.L., Madrid, Spain) was used for the statistical analysis. Before statistical analysis data were checked for normality by using Shaipro Wilk test. Categorical variables were analysed using the chi square test or Fisher's exact test as appropriate. Kappa coefficient was also used to find the agreement between HPE, direct microscopy and culture variables. For all statistical tests, $\mathrm{p}<0.05$ was considered to indicate a significant difference. All tests of statistical significance were two tailed.

\section{Results}

Of the suspected cases of FRS, a total of 25 cases were suspected to have IFRS based on clinical history, nasal endoscopic and radiological evidences and were confirmed by microbiological and HPE. AIFRS was seen in $56 \%$ cases, CIFRS in $36 \%$ cases and rest $8 \%$ had CGFRS The age of the patients ranged from 20 years to 75 years, sex ratio (male: female) being 1:1.27 (Table 1). 64\% cases were educated and $60 \%$ were employed.

\begin{tabular}{|c|c|c|c|c|c|c|}
\hline Symptom & $\begin{array}{l}\text { AIFRS } \\
(n=14)\end{array}$ & $\begin{array}{l}\mathrm{p}- \\
\text { value }\end{array}$ & $\begin{array}{l}\text { CIFRS } \\
(n=9)\end{array}$ & $\begin{array}{l}\mathrm{p}- \\
\text { value }\end{array}$ & $\begin{array}{l}\text { CGFRS } \\
(n=2)\end{array}$ & $\begin{array}{l}p- \\
\text { value }\end{array}$ \\
\hline \multirow[t]{2}{*}{ Duration (mean) } & 15 days & 0.00 & $\begin{array}{l}2.21 \\
\text { years }\end{array}$ & 0.283 & 1 year & 0.8 \\
\hline & $\mathrm{n}(\%)$ & $\begin{array}{l}\mathrm{p}- \\
\text { value }\end{array}$ & $\mathrm{n}(\%)$ & $\begin{array}{l}\mathrm{p}- \\
\text { value }\end{array}$ & $\mathrm{n}(\%)$ & $\begin{array}{l}\mathrm{p}- \\
\text { value }\end{array}$ \\
\hline Nasal obstruction & $8(57.14)$ & 0.00 & $7(77.77)$ & 0.61 & $2(100)$ & 1.00 \\
\hline Headache & $\begin{array}{l}10 \\
(71.42)\end{array}$ & 0.37 & $4(44.44)$ & 0.30 & $2(100)$ & 0.52 \\
\hline Nasal discharge & $2(14.28)$ & 0.00 & $5(55.55)$ & 1.00 & $2(100)$ & 0.49 \\
\hline Smell disturbances & $17.14)$ & 0.05 & 0.00 & - & $1(50)$ & 0.52 \\
\hline Loss of vision & $\begin{array}{l}10 \\
(71.42)\end{array}$ & 0.00 & $1(11.11)$ & 0.67 & 0.00 & - \\
\hline Sneezing & 0.00 & - & 0.00 & - & 0.00 & - \\
\hline Proptosis & $3(21.42)$ & 0.70 & 0.00 & - & $1(50)$ & 0.32 \\
\hline Fever & $6(42.85)$ & 0.00 & 0.00 & - & 0.00 & - \\
\hline Postnasal drip & 0.00 & - & 0.00 & - & $1(50)$ & 0.19 \\
\hline Facial swelling & $2(14.28)$ & 0.59 & $1(11.11)$ & 0.58 & 0.00 & - \\
\hline CNS symptoms & $5(35.71)$ & 0.00 & 0.00 & - & 0.00 & - \\
\hline Diplopia & $1(7.14)$ & 1.00 & 0.00 & - & 0.00 & - \\
\hline
\end{tabular}

\begin{tabular}{|c|c|c|c|c|c|c|}
\hline Epistaxis & 0.00 & - & 0.00 & - & 0.00 & - \\
\hline Facial pain & $1(7.14)$ & 0.37 & $1(11.11)$ & 0.25 & 0.00 & - \\
\hline $\begin{array}{l}\text { Ocular/nasal } \\
\text { itching }\end{array}$ & 0.00 & - & 0.00 & - & 0.00 & - \\
\hline \multicolumn{7}{|l|}{ Co- morbidities } \\
\hline Diabetes mellitus & $9(64.2)$ & 0.00 & $2(22.2)$ & 0.68 & 0.00 & - \\
\hline Hypertension & $8(57.1)$ & 0.00 & $1(11.1)$ & 0.59 & 0.00 & - \\
\hline Bronchial asthma & $4(28.5)$ & 0.11 & 0.00 & - & 0.00 & - \\
\hline $\begin{array}{l}\text { Previous nasal } \\
\text { surgeries }\end{array}$ & 0.00 & - & 0.00 & - & 0.00 & - \\
\hline Tuberculosis & $1(7.14)$ & 0.67 & 0.00 & - & 0.00 & - \\
\hline Any Allergy & 0.00 & - & 0.00 & - & 0.00 & - \\
\hline Carcinoma & 0.00 & - & 0.00 & - & $1(50)$ & 0.00 \\
\hline Psychiatric illness & 0.00 & - & 0.00 & - & 0.00 & - \\
\hline $\begin{array}{l}\text { Immuno- } \\
\text { compromised }\end{array}$ & $9(64.28)$ & 0.00 & $2(22.2)$ & 0.67 & $1(50)$ & 0.34 \\
\hline
\end{tabular}

Table 1: Clinical presentations of the patients.

The mean duration of AIFRS was 15 days among our cases, while it was 2.21 years in CIFRS. A wide range of symptoms was noticed ranging from non-specific symptoms of rhinosinusitis like nasal obstruction, nasal discharge to symptoms indicating invasiveness like loss of vision, proptosis and neurological symptoms. Headache (71.42\%) and loss of vision (71.42\%) were common symptoms among AIFRS cases whereas nasal obstruction, nasal discharge and headache were common in CIFRS cases. CNS symptoms like focal neurological deficit, cranial nerve palsies etc. were seen in $35.7 \%$ of AIFRS.

$48 \%$ cases were immunocompromised, diabetes mellitus being the most common cause, seen in $64.2 \%$ AIFRS and $22.2 \%$ CIFRS cases. Hypertension was next common co-morbidity being present in $57.1 \%$ AIFRS and $11.1 \%$ CIFRS cases. Bronchial asthma was seen in 4 cases and tuberculosis in one case of AIFRS. One of the two CGFRS patients had carcinoma too. The immunocompromised status was seen among IFRS cases, being present in $64.2 \%$ AIFRS, 50\% CGFRS and $22.2 \%$ CIFRS.

On nasal endoscopy, black crusts were seen in the majority of patients (56\%). Other findings seen were perforation of septum, polyps-unilateral or bilateral, nasal septum deviation and hypertrophy the inferior turbinates. Polyps were commonly seen in chronic invasive cases $(88.8 \%)$ whereas perforation of septum was more associated with AIFRS (35.71\%).

CT or MRI scans were performed in all these 25 patients. Evidence of invasion like bone erosion and intracranial involvement were seen in 12 patients. Figure 1 shows heterogenous opacities in the right nasal cavity, maxillary and ethmoidal sinuses. Heterogeneous opacities were seen more in chronic cases than acute cases. Calcification was seen only in CIFRS (33.3\%). Bone erosion, intracranial or intra-orbital extensions and mucosal thickening were more common in AIFRS than 
Citation: Kaur R, Lavanya S, Khurana A, Gulati A, Dhakad MS (2016) Invasive Fungal Rhinosinusitis: An Observational Study in an Indian Tertiary Care Hospital. Lung Dis Treat 2: 109. doi:10.4172/2472-1018.1000109

Page 3 of 7

chronic ones. Figure 2 shows, a patient with periorbital cellulitis and CT scan of the same patient of the effected eye.

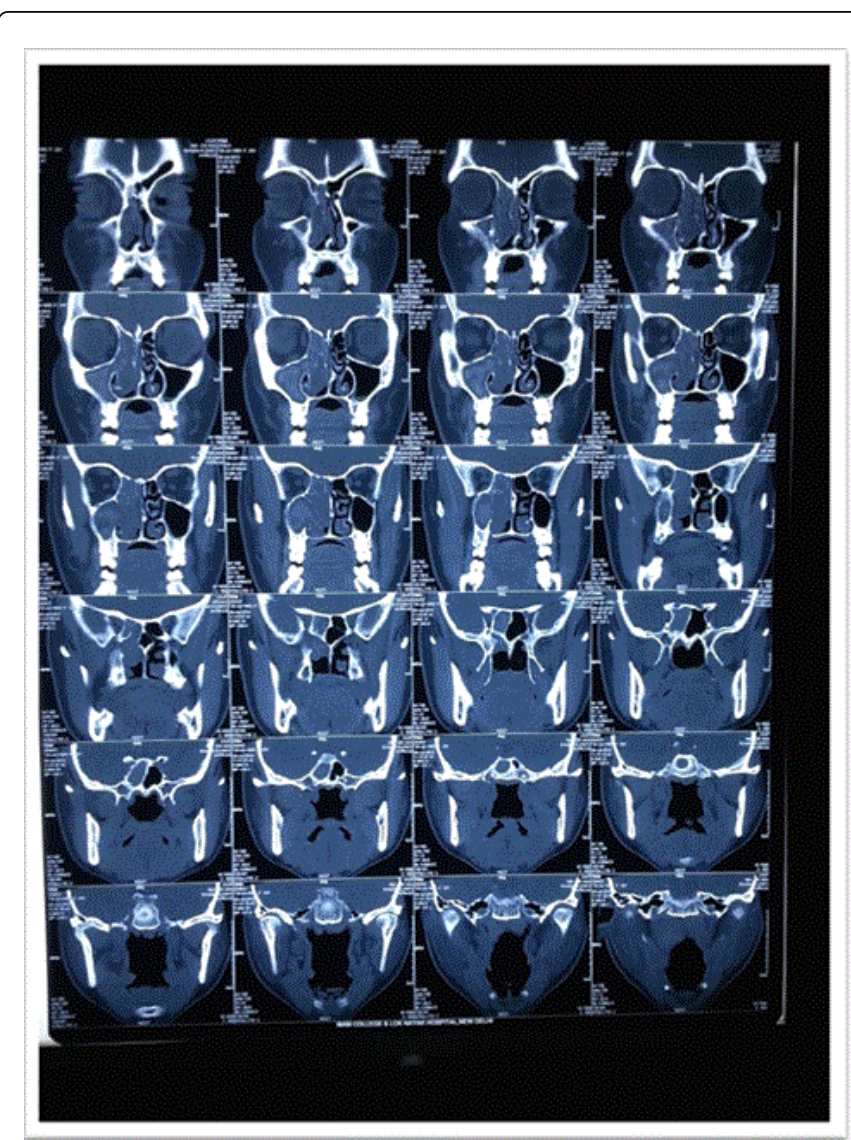

Figure 1: Non contrast CT scan, coronal section of FRS patient showing heterogenous opacities in the right nasal cavity, maxillary and ethmoidal sinuses.

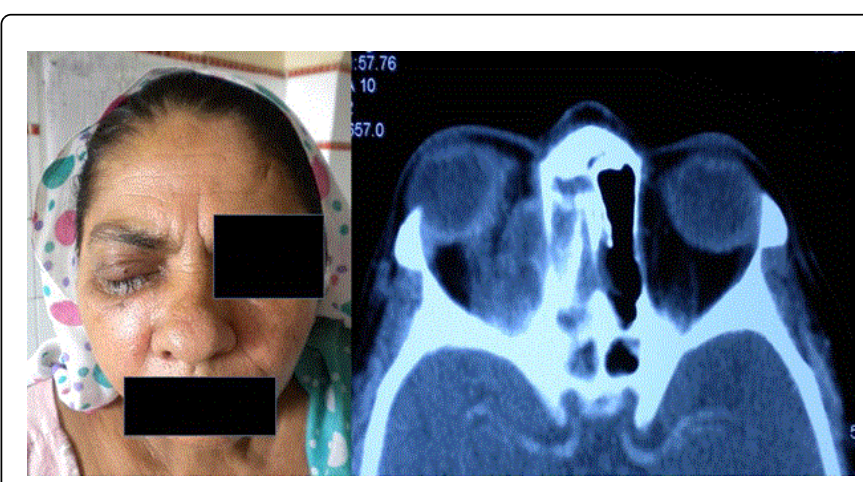

Figure 2: Invasive fungal Rhinosinusitis. (A) A patient with periorbital cellulitis. (B) Computed tomography of the same patient shows dense area in the effected eye.

HPE revealed necrosis of submucosa, bone, vascular tissue in $96 \%$ cases. Aseptate fungal hyphae were present in $78.5 \%$ of AIFRS and in $77.7 \%$ cases of CIFRS. Septate hyphae were seen in a lesser number of cases being present in $28.5 \%$ of AIFRS and $11.1 \%$ of CIFRS. Both the patients of CGFRS had evidence of granulation tissue and one of them had presence of septate fungal hyphae on HPE. PAS stained nasal biopsy sample shows fungal hyphae indicated by arrows in Figure 3.

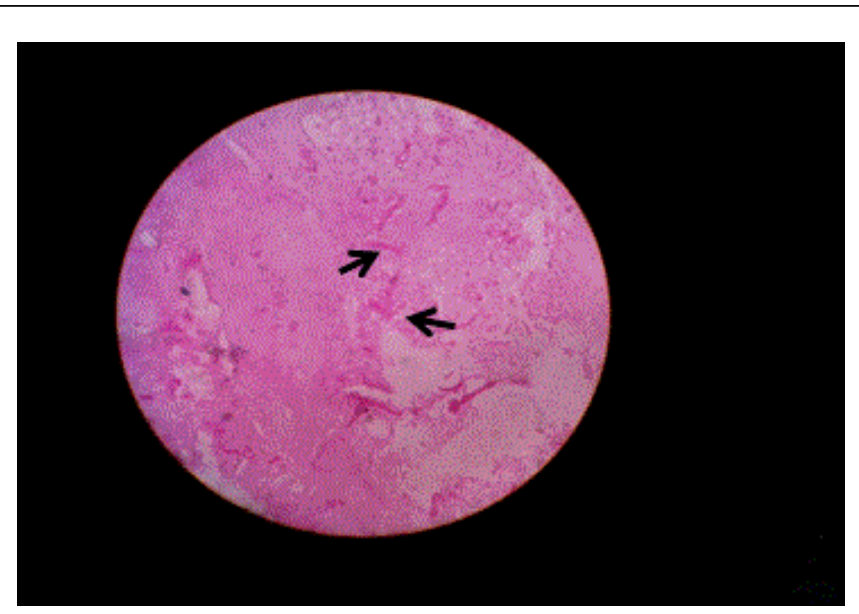

Figure 3: PAS staining of nasal biopsy sample showing fungal hyphae (arrows) at 40X.

On microbiological examination, fungal elements were seen by microscopy in majority of AIFRS cases (92.8\%) but only half of the chronic cases had evidence of fungal hyphae. On culture, Rhizopus arrhizus (64.2\%) was the most common isolate (Figure 4) among AIFRS cases followed by Aspergillus flavus (35.7\%). Mucor species were solely isolated from AIFRS (14.3\%). Whereas in chronic cases Aspergillus flavus (44.4\%) seemed to be the major isolate. Other isolates identified from CIFRS were Aspergillus niger, Alternaria spp., Penicillium spp. and Candida albicans. Two cases of CGFRS yielded Aspergillus flavus and Penicillium spp.

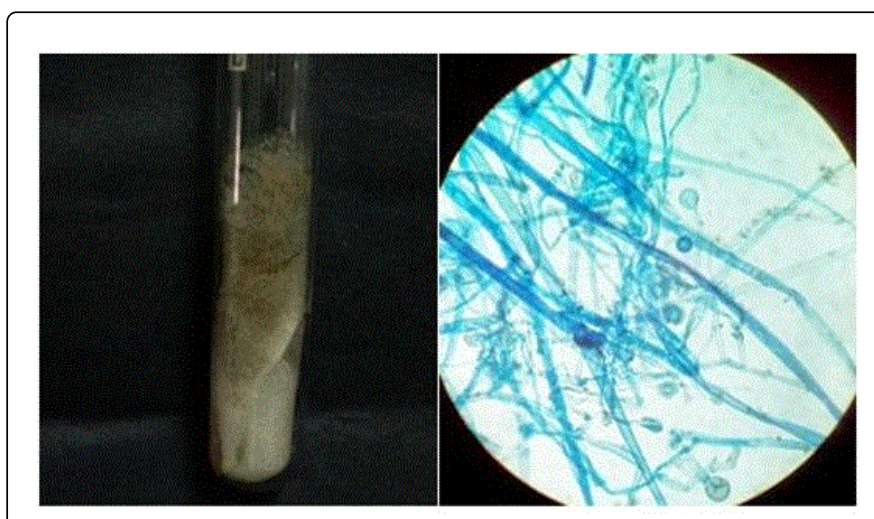

Figure 4: Isolate of Rhizopus arrhizus on Sabouraud's dextrose agar and Lactophenol cotton blue mount.

Surgical intervention like debridement and functional endoscopic sinus surgery (FESS) was done in all the invasive cases. Amphotericin $\mathrm{B}$ was given to $71.4 \%$ of AIFRS and $33.3 \%$ of CIFRS. Itraconazole was given to $21.4 \%$ of AIFRS, $55.5 \%$ of CIFRS and both the cases of CGFRS depending on the histopathological and microbiological report. Two patients expired despite initiation of the therapy; both the patients were cases of AIFRS with mucormycosis and duration of less than 2 weeks. 
$55 \%$ agreement was seen between culture and direct microscopy in CIFRS cases, while in AIFRS cases, $2.5 \%$ agreement was seen between culture and direct microscopy, $4.58 \%$ between culture and HPE and $9 \%$ between direct microscopy and HPE. The sensitivity of the different detection techniques was seen to be $46.37 \%$ by HPE, $68.11 \%$ by direct microscopy by $\mathrm{KOH} \&$ calcofluor white mount and $86.95 \%$ by fungal culture but the specificity \& positive predictive value of all techniques were $100 \%$. The negative predictive value ranged from $13.95 \%$ (HPE) and $21.42 \%$ (direct microscopy) to $40 \%$ (culture) as shown in Table 2.

\begin{tabular}{|l|l|l|l|l|}
\hline Methods & Sensitivity & Specificity & $\begin{array}{l}\text { Positive } \\
\text { predictive } \\
\text { value }\end{array}$ & $\begin{array}{l}\text { Negative } \\
\text { predictive } \\
\text { value }\end{array}$ \\
\hline Culture & $86.95 \%$ & $100 \%$ & $100 \%$ & $40 \%$ \\
\hline Direct microscopy & $68.11 \%$ & $100 \%$ & $100 \%$ & $21.42 \%$ \\
\hline HPE & $46.37 \%$ & $100 \%$ & $100 \%$ & $13.95 \%$ \\
\hline
\end{tabular}

Table 2: Correlation of culture, direct microscopy and HPE with FRS cases.

\section{Discussion}

There has been an unprecedented increase in the number and variety of fungal paranasal sinus disease reports in the past two decades. Stressing on the need for classification of AFRS for timely and effective treatment and prediction of prognosis in most cases [14]. Deshazo et al. [4] proposed a new classification for tissue IFRS based on the clinical condition, immune status, histopathology, and fungus infection: AIFRS, CGFRS and CIFRS types. With a disease course of $<4$ weeks is very typically seen in AIFRS cases in an immunocompromised setting [3]. While a time course of $>12$ weeks along with dense accumulation of hyphae with occasional vascular invasion and sparse inflammatory reaction along with involvement of local structure are typical of CIFRS cases [15]. In CGFRS, a granulomatous response with considerable fibrosis is seen [14].

Among the patients of AIFRS, the age range was 22-75 years with a mean age of 54 years and sex ratio (M: F) of 1:1.33. In a study on IFRS in Thailand over a period of 10 years from 1998 to 2008, male: female ratio was also reported to be $1: 1.25$, with an average age being 52.27 years (range: 16-78) [5]. Similar to our study, Montone et al. [16] in USA found the mean age to be 54 years (24-82) but the M: F ratio was 1.5:1. Among CIFRS, the age range was $20-63$ years, mean age being 45 years and sex ratio (M:F) 1:2, and both the cases of CGFRS were males, aged 42 years. Similar mean age and age range was found in a western study was 48 years (21-65) in CIFRS cases while a higher age of 58 years (50-66) was noted in CGFRS cases [16]

It has been seen that patients of CIFRS present clinically with an enlarging mass in cheek, orbit, nose and paranasal sinus regions. With intracranial extension there may be symptoms like headache, localizing neurological findings, seizures, proptosis, facial pain etc. While acute fulminant type presents with fever and headache in initial stages and proptosis, blindness, conjugal chemosis, opthalmoplegia, signa and symptoms of meningeal involvement, cerebral infarction, multiple cranial nerve palsies on invasion to different sites [7].

The most common symptoms in AIFRS cases were headache and loss of vision, $71.42 \%$ each followed by nasal obstruction $(57.14 \%)$, fever (42.85\%) and CNS symptoms (35.7\%) with a mean duration of symptoms being 15 days. This was similar to the study done by Piromchai in Thailand, 2008 who also found headache (59.3\%) to be the most common symptom, followed by visual loss (47.5\%), facial pain (35.6\%) and fever (33.9\%) [5].

Even though majority of our FRS patients were immunocompetent (81.15\%), immunocompromised state was seen to be statistically significant in AIFRS cases. AIFRS usually occurs in immunocompromised cases as described by DeShazo et al. [4]. In our study, the immunocompromised status was seen among IFRS cases, being present in $64.2 \%$ AIFRS, 50\% CGFRS and $22.2 \%$ CIFRS. In a study in Thailand in 2008, it was seen that patients with immunocompromised status had significant greater risk for acute than CIFRS similar to our study [5]. In another study in USA in 2008, 100\% of AIFRS cases were immunosuppressed [16]. A significant proportion of the Indian population lives below the poverty line and hence may be malnourished [17]. Although they are not "immunocompromised" in the classical sense, their poor nutritional status may render them more susceptible to invasive disease [17].

Diabetes mellitus was the most common co-morbidity among AIFRS patients being present in $64.2 \%$ cases and hypertension was seen in $57.1 \%$ cases. The associated co-morbidities with AIFRS as seen in our cases were similar to a study in Thailand (2008) which found diabetes to be associated in $66.6 \%$ of their AIFRS cases. However, Pagella et al. [18] reported in AIFRS cases haemtological malignancies represented the principal comorbidity (100\%) and Montone et al. [14] from USA also found haematological disorders (84\%) to be more commonly associated with AIFRS patients $[5,8]$. Also Micheal et al. [17] had an association of diabetes in $62.7 \%$ of AIFRS cases in their study. In CIFRS cases, diabetes was present in $22.2 \%$ and hypertension in $11.1 \%$ cases [17]. While Pagella et al. [18] reported in chronic form diabetes mellitus (87.5\%) to be the principal comorbidity. Micheal et al. [17] in Tamilnadu found underlying co-morbidities in 53\% of CIFRS cases when compared to $90.3 \%$ in AIFRS forms which included $62.7 \%$ cases of diabetes.

In our study, bone erosions, intraorbital and intracranial extension, mucosal thickening and heterogenous opacities on bilateral sides were significantly associated and commonly seen in AIFRS cases. Homogenous opacities on unilateral side were also significantly associated with fungal ball cases in our study, showing their limitation to one sinus as described in a review of imaging studies of FRS cases. Other findings like heterogenous opacities, mucosal thickening and calcifications are also documented features of fungal ball [9].

Heterogenous opacities on bilateral sides were significant findings in our cases of AFRS (60\%) proving the tendency of the disease to be bilateral in nature [9]. Heterogeneous opacities were the commonest finding in CIFRS cases followed by mucosal thickening and calcification. The CT findings seen in a study in IFRS cases by workers in Thailand, 2008 were also sinus wall erosion, mucosal thickening and air fluid levels [5]. The features of CGFRS are similar to CIFRS cases in our study quite in concordance to the features described by Aribandi et al. [9] in his review in which a hyperattenuating soft tissue collection is seen at non contrast CT within one or more sinuses in CIFRS cases. Workers in Brazil in 2002 also found significant associations of bone erosions, microcalcification and mucosal thickness with AFRS cases [1]. Bone erosion was again significantly associated with AFRS in a study done in Chandigarh (India) in 2002-2003 [19].

On HPE of our cases, necrosis of submucosa, bone, and vascular tissue were seen in $100 \%$ cases of AIFRS and $78.57 \%$ had evidence of 
Citation: Kaur R, Lavanya S, Khurana N, Gulati A, Dhakad MS (2016) Invasive Fungal Rhinosinusitis: An Observational Study in an Indian Tertiary

Page 5 of 7

aseptate fungal hyphae in them. Both were found to be statistically significant $(\mathrm{p}<0.001)$ (Table 3$)$. Septate fungal hyphae were seen in $28.57 \%$ cases and acute inflammation in only one patient. Similar findings were seen in chronic invasive cases too. These features were similar to the histopathological findings described in literature, as hyphal invasion of blood vessels, vasculitis with thrombosis, haemorrhage, tissue infarction and neutrophilic infiltration are commonly seen in AIFRS cases and same features with low grade inflammation are seen to appear in CIFRS cases [4].

\begin{tabular}{|c|c|c|c|c|c|c|}
\hline & \multicolumn{2}{|l|}{ AIFRS } & \multicolumn{2}{|l|}{ CIFRS } & \multicolumn{2}{|l|}{ CGFRS } \\
\hline \multirow[t]{2}{*}{ Nasal endoscopy findings } & \multicolumn{2}{|l|}{$(n=14)$} & \multicolumn{2}{|l|}{$(n=9)$} & \multicolumn{2}{|l|}{$(n=2)$} \\
\hline & $\mathrm{n}(\%)$ & $p$-value & $\mathrm{n}(\%)$ & $\mathrm{p}$-value & $\mathrm{n}(\%)$ & $p$-value \\
\hline Polyp-unilateral & 0 & - & $5(55.5)$ & 0.235 & 0 & - \\
\hline Polyp-bilateral & $1(7.14)$ & 0.019 & $3(33.3)$ & 1 & $1(50)$ & 0.611 \\
\hline Deviated nasal septum & $1(7.14)$ & 0.176 & $1(11.1)$ & 0.462 & $1(50)$ & 0.289 \\
\hline Secretions/crusts-black & $13(92.85)$ & 0 & $1(11.1)$ & 0.407 & 0 & - \\
\hline Secretions-greenish yellow & 0 & - & $1(11.1)$ & 0.757 & 0 & - \\
\hline Inferior turbinate hypertrophy & $2(14.28)$ & 0.724 & $1(11.1)$ & 0.961 & $1(50)$ & 0.085 \\
\hline Perforation of septum & $5(35.71)$ & 0 & 0 & - & 0 & - \\
\hline Middle turbinate hypertrophy & 0 & - & 0 & - & 0 & - \\
\hline \multicolumn{7}{|l|}{ Radiological findings } \\
\hline Heterogenous opacities-unilateral & $2(14.28)$ & 0.113 & $3(33.33)$ & 0.92 & $1(50)$ & 0.5769 \\
\hline Heterogenous opacities-bilateral & $1(7.14)$ & 0.003 & $4(44.4)$ & 0.874 & $1(50)$ & 0.816 \\
\hline Homogenous opacities-unilateral & $1(7.14)$ & 0.565 & 0 & - & 0 & - \\
\hline Homogenous opacities-bilateral & 0 & - & $1(11.1)$ & 0.009 & 0 & - \\
\hline Mucosal thickening & $8(57.14)$ & 0.0342 & $3(33.3)$ & 1 & 0 & - \\
\hline Bone erosion & $10(71.42)$ & 0.003 & $1(11.1)$ & 0.077 & $1(50)$ & 0.715 \\
\hline Intracranial/intraorbital extension & $10(71.42)$ & 0 & $1(11.1)$ & 0.204 & $1(50)$ & 0.506 \\
\hline Calcification & 0 & - & $3(33.3)$ & 0 & 0 & - \\
\hline \multicolumn{7}{|l|}{ Histopathological findings } \\
\hline Acute inflammation & $1(7.14)$ & 0.676 & $1(11.1)$ & 0.918 & 0 & - \\
\hline Chronic inflammation & 0 & - & 0 & - & $1(50)$ & 0.001 \\
\hline Granulation tissue & 0 & - & 0 & - & $2(100)$ & 0.002 \\
\hline Allergic mucin & 0 & - & 0 & - & 0 & - \\
\hline Fungal hyphae-septate & $4(28.57)$ & 0.702 & $1(11.1)$ & 0.312 & $1(50)$ & 0.398 \\
\hline Fungal hyphae aseptate & $11(78.57)$ & 0 & $7(77.7)$ & 0 & 0 & - \\
\hline Necrosis of submucosa, bone, vascular tissue & $14(100)$ & 0 & $9(100)$ & 0 & $1(50)$ & 0.646 \\
\hline Calcification & 0 & - & 0 & - & 0 & - \\
\hline Carcinoma & 0 & - & 0 & - & 0 & - \\
\hline \multicolumn{7}{|l|}{ Microbiological examination } \\
\hline Direct microscopy $(n=47)$ & $13(92.85 \%)$ & 0.05 & $5(55.55 \%)$ & 0.628 & $1(50 \%)$ & 0.832 \\
\hline Septate hyphae & $2(14.28 \%)$ & 0.101 & $4(44.44 \%)$ & 0.826 & $1(50 \%)$ & 0.476 \\
\hline
\end{tabular}




\begin{tabular}{|c|c|c|c|c|c|c|}
\hline Aseptate hyphae & $8(57.14 \%)$ & 0 & $1(11.11 \%)$ & 0.594 & 0 & - \\
\hline Both septate and aseptate hyphae & $3(21.43 \%)$ & 0.03 & 0 & - & 0 & - \\
\hline Budding yeast cells & 0 & - & 0 & - & 0 & - \\
\hline Culture $(n=60)$ & $14(100 \%)$ & 0.187 & 5 (55.55\%) & 0.013 & $2(100 \%)$ & 1 \\
\hline Isolates & $\begin{array}{l}\text { A. flavus }(5 / 35.7 \%) \\
\text { A. niger }(1 / 7.14 \%) \\
\text { R. arrhizus } \\
\text { (9/64.2\%) } \\
\text { Mucor spp. } \\
(2 / 14.3 \%)\end{array}$ & & $\begin{array}{l}\text { A. flavus }(4 / 44.4 \%) \\
\text { A. niger }(1 / 11.1 \%) \\
\text { Alternaria spp. } \\
(1 / 11.1 \%) \\
\text { Penicillium spp. } \\
(1 / 11.1 \%) \text { C. albican } \\
(1 / 11.1 \%)\end{array}$ & & $\begin{array}{l}\text { A. flavus } \\
(1 / 50 \%) \\
\text { Penicillium } \\
\text { spp. (1/50\%) }\end{array}$ & \\
\hline
\end{tabular}

Table 3: Investigative profile of the patients of Invasive fungal rhinosinusitis.

In the two cases of CGFRS, granulation tissue was seen in both the cases with presence of septate hyphae in one of them. Necrosis of submucosa, bone, and vascular tissue and chronic inflammation were also seen in one case. These findings were similar to a study by Montone et al. [16] in USA over 1991-2008 where granulomatous inflammation and chronic inflammation were also seen in their CGFRS cases.

Our nasal endoscopy findings were nearly similar to a study in Pondicherry in 2009 where polyps were the commonest findings, bilateral polyps seen in $47.76 \%$ and unilateral in $17.9 \%$. Purulent nasal discharge being seen in middle meatus in $19.4 \%$, black crust seen only in $1.49 \%$ cases with the remaining patients having osteo-meatal complex obstruction due to deviated nasal septum, concho bullosa etc. [14]. Black crust and perforation of septum were significantly seen in AIFRS along with polyps on bilateral side. Piromachai from Thailand in 2008 also noticed mucosal necrosis and black crust as the most common finding in their cases of AIFRS [5].

Polyps were significantly associated with AFRS cases in our study similar to a study done in Brazil in 2002 [19]. 90\% of their patients were having AFRS and fungal ball, with nasal endoscopic examination showing secretions in majority of their FRS patients (91.9\%) with yellow secretions being predominant followed by green secretions. Polyps were seen in $40.3 \%$ cases including bilateral in $16.1 \%$ and unilateral polyps in $24.1 \%$, inferior turbinate hypertrophy was seen in $27.4 \%$ and adenoid hypertrophy in $4.8 \%$ cases. Our study also showed similar observations, in addition middle turbinate hypertrophy being significantly associated in our AFRS cases. Polyps on unilateral side $(100 \%)$ and greenish yellow secretions (50\%) were seen in majority of our fungal ball cases.

Direct microscopy with $\mathrm{KOH}$ mount was positive in 19 cases (76\%); 92.85\% of AIFRS cases which was statistically significant, $55.55 \%$ of CIFRS, $50 \%$ of CGFRS cases. Culture was positive in 100\% of AIFRS cases with the isolation of Rhizopus arrhizus as the commonest isolate, in concurrence to different studies from India, where Rhizopus arrhizus was the most common isolate among AIFRS cases [17,20]. However, Prateek et al. [10] found Mucor spp. (100\%) as the common isolate among AIFRS cases. But in a western study from USA in 2008 by Montone et al. [16], Aspergillus spp. (49\%) were the most common isolate followed by Rhizopus spp. (33\%).

$55.55 \%$ of CIFRS cases were positive for fungi by culture, yielding various isolates like Aspergillus spp., Penicillium spp., Alternaria spp. and Candida albicans similar to the studies by Micheal et al. [17] in
Tamilnadu and Das et al. [20] in Chandigarh, India, where Aspergillus flavus was the common isolate. Aspergillus spp. also commonest isolates in a study by Prateek et al. [10] in a tertiary care hospital in Uttar Pradesh, India, Aspergillus fumigatus (66.6\%) being the most common followed by A. flavus (33.37\%). However, in USA in 2008, out of 4 patients having CIFRS, Candida albicans was isolated in one and $S$. apiospermium in another. Culture was not performed in the rest 2 cases [16]. Another study in Thailand done during 1998-2008 reported Rhizopus spp. to be the commonest isolate followed by Aspergillus spp. in both types of IFRS [5].

In our study, culture and direct microscopy (by $\mathrm{KOH}$ mount, calcofluor white and Grams) showed agreement in almost 20\% of cases. The percentage of agreement between histopathological examination and direct microscopy was only $12.6 \%$, whereas not much agreement was seen between histopathological examination and culture in our study.

Out of the 2 CGFRS cases in our study, Aspergillus flavus was isolated from one case and Penicillium spp. from the other quite similar to a Indian study [10], where A. flavus (100\%) was isolated from both the cases of CGFRS and also to another Indian study by Das et al. [20], with $A$. flavus being the commonest isolate. These results were also in concordance to a western study from USA in 2008 where the culture grew A. flavus from both the cases of CGFRS [16]. The variation in isolates noticed among the eastern and western countries might be attributed to different geographical features and climatic conditions.

According to Chakrabarti et al. [21], treatment for AIFRS and CIFRS is radical debridement and antifungal agent while for CGFRS is debridement, aeration and itraconazole. In our study, $100 \%$ cases of AIFRS, CIFRS and CGFRS underwent surgical debridement and itraconazole drug was given per orally in $100 \%$ cases of CGFRS, $55.5 \%$ of CIFRS and $21.4 \%$ of AIFRS while amphotericin B drug was given through parenteral route in $71.4 \%$ of AIFRS and $33.3 \%$ of CIFRS cases. The outcome was favorable in majority of our cases, they improved and were discharged on medication. Only 2 patients expired both being AIFRS cases even though treatment was started early in these case on presumptive diagnosis. Rhizopus arrhizus was the causative agent in both of them. It has been reported earlier that the diagnosis is usually poor in invasive cases if the treatment is not started early [4]. In a case series, 49 patients with rhinocerebral mucormycosis, the mortality was $70 \%$ in cases treated with antifungal agents alone versus $14 \%$ in cases treated with antifungal agents plus surgery [22]. 
Citation: Kaur R, Lavanya S, Khurana N, Gulati A, Dhakad MS (2016) Invasive Fungal Rhinosinusitis: An Observational Study in an Indian Tertiary

Prompt diagnosis and initiation of appropriate therapy are essential to avoid a protracted or fatal outcome. Surgery is important both in the diagnosis and debridement of necrotic tissue as well improving aeration and drainage within the sinuses. This is often done endoscopically to preserve the normal anatomy wherever possible $[8,15]$. Amphotericin B, $1-1.5 \mathrm{mg} / \mathrm{kg} /$ day for a total dose of 2 grams or more is the gold standard antifungal therapy. Voriconazole is the treatment of choice for invasive aspergillosis rhinosinusitis [23].

The outcome was favourable in majority of our invasive cases as they improved and were discharged on medication. Only 2 patients expired both being AIFRS cases even though treatment was started early in these case on presumptive diagnosis. Rhizopus arrhizus was the causative agent in both of them. It has been reported earlier that the diagnosis is usually poor in invasive cases if the treatment is not started early [4].

In conclusion, prompt diagnosis and early initiation of treatment is the key to reduce morbidity and mortality in invasive fungal rhinosinusitis cases. Radiological features help in presumptive diagnosis of FRS. Histopathology and direct microscopy give a clue to presence of fungi and the culture confirms the etiological agent. Emphasis must be laid on direct microscopy as it directs towards confirmation of diagnosis and early initiation as well as choice of treatment.

\section{References}

1. Dall'Igna C, Palombini BC, Anselmi F, Araujo E, Dall'Igna DP (2005) Fungal rhinosinusitis in patients with chronic sinonasal disease. Braz J Otorhinolaryngol 71: 712-720.

2. Chakrabarti A, Das A, Panda NK (2009) Controversies surrounding the categorization of fungal sinusitis. Medical Mycology 47: S299-S308.

3. Chakrabarti A, Denning DW, Ferguson BJ, Ponikau J, Buzina W, et al. (2009) Fungal Rhinosinusitis: A Categorization and Definitional schema addressing current controversies. Laryngoscope 119: 1809-1018.

4. deShazo RD, Chaplin K, Swain RE (1997) Fungal sinusitis. N Engl J Med 337: 254259.

5. Piromchai P, Thanaviratananich S (2012) Acute versus Chronic invasive fungal rhinosinusitis: a case-control study. Infect dis: Research and Treatment 5: 43-48.

6. Chen CY, Sheng WH, Cheng A, Chen YC, Tsay W, et al. (2011) Invasive fungal sinusitis in patients with hematological malignancy: 15 years' experience in a single university hospital in Taiwan. BMC Infectious Diseases 11: 1-9.

7. Chakrabarti A, Sharma SC (2000) Paranasal sinus mycoses. Indian J Chest Dis Allied Sci 42: 293304
8. Bazan C, Rinaldi MG, Rauch RR, Jinkins JR (1991) Fungal infections of the brain. Neuroimaging Clin N Am 1: 57-88.

9. Aribandi M, McCoy VA, Bazan C (2007) Imaging features of invasive and non-invasive fungal sinusitis: a review. Radiographics 27: 1283-1296.

10. Prateek S, Banerjee G, Gupta P, Singh M, Goel MM, et al. (2013) Fungal rhino sinusitis: A prospective study in a University hospital of Uttar Pradesh. Indian J Med Microbiol 31: 266-269.

11. Moore GS, Jaciow DM (1979) Mycology for the Clinical Laboratory. Reston, VA: Prentice-Hall.

12. Forbes BA, Sahm DF, Weissfeld AS (2002) Laboratory methods in basic mycology. In Bailey and Scott's Diagnostic Microbiology (11th edn.) St Louis: Mosby.

13. Koneman EW, Allen SD, Janda WM, Schreckenberger PC (1997) Mycology. In Color Atlas and Textbook of Diagnostic Microbiology (5th edn.) Philadelphia, PA: Lippincott Williams \& Wilkins.

14. Karthikeyan P, Coumare VN (2010) Incidence and presentation of fungal sinusitis in patient diagnosed with chronic rhinosinusitis. Indian J Otolaryngol Head Neck Surg 62: 381-385.

15. Chatterjee SS, Chakrabarti A (2009) Epidemiology and Medical Mycology of Fungal Rhinosinusitis. Otorhinolaryngology Clinics: An International Journal 1: 1-13.

16. Montone KT, Livoisi VA, Feldman MD, Palmer J, Chiu AG, et al. (2012) Fungal Rhinosinusitis: A Retrospective Microbiologic and Pathologic Review of 400 Patients at a Single University Medical Center. International J Otolaryngol 122: 1438-1445.

17. Michael RC, Michael JS, Ashbee RH, Mathews MS (2008) Mycological profile of fungal sinusitis: An audit of specimens over a 7-year period in a tertiary care hospital in Tamil Nadu. Indian J Pathol Microbiol 51: 493-496.

18. Pagella F, De Bernardi F, Dalla Gasperina D, Pusateri A, Matti E, et al. (2016) Invasive fungal rhinosinusitis in adult patients: Our experience in diagnosis and management. J Craniomaxillofac Surg 44: 512-520.

19. Saravanan K, Panda NK, Chakrabarti A, Das A, Bapuraj RJ (2006) Allergic fungal rhinosinusitis: an attempt to resolve the diagnostic dilemma. Arch Otolaryngol Head Neck Surg 132: 173-178.

20. Das A, Bal A, Chakrabarti A, Panda NK, Joshi K (2009) Spectrum of fungal rhinosinusitis; Histopathologist's perspective. Histopathology 54: 854-859.

21. Chakrabarti A, Das A, Panda NK (2004) Overview of Fungal Rhinosinusitis. Indian J Otolaryngology and Head and Neck Surgery 56: 251-258.

22. Hathiram BT, Khattar VS (2009) Surgical Management of Fungal Rhinosinusitis. Otorhinolaryngology Clinics: An International Journal 1: 69-75

23. Herbrecht R, Denning DW, Patterson TF, Bennett JE, Greene RE, et al. (2002) Voriconazole versus amphotericin B for primary therapy of invasive aspergillosis. N Engl J Med 347: 408-415. 